\title{
Anterior dislocation following primary total hip replacement
}

\author{
Bernardino Saccomanni
}

Received: 7 November 2008 / Published online: 9 December 2008

(C) Springer-Verlag 2008

Dear Editor,

It was interesting to read the article by $\mathrm{Ng}$ et al. in a issue of the Hong Kong Journal of Orthopaedic Surgery [1]. The authors have described a review of five patients with anterior dislocation of the hip after having undergone a total hip replacement by the posterior approach.

While it is accurately mentioned in the article that it is rare to find anterior dislocation after using the posterior approach for total hip arthroplasty, I fail to understand why the article is titled 'aetiology and treatment' because the described aetiology is nothing but a complication of excessive anteversion, which is well known. The cause of three of the reported five cases was established by fluoroscopy. It would be of interest to other readers also to know why such a definitive aetiological factor was not investigated for the two other patients, especially when such cases are reported. Also, I would like to highlight that, in all these reported cases, the approach had nothing to do with the type of dislocation (anterior). Rather, as the authors themselves mention, the anterior dislocations were only because of the high angle of anteversion, which was wrongly done during the surgery. I appreciate the fact that authors have reported their avoidable complications as a scientific paper. The rehabilitation and treatment protocol after any such adversity is nicely described in this article and is quite informative.

\section{Reference}

1. Ng TP, Yau WP, Tang WM, Chiu KY (2003) Anterior dislocation following primary total hip replacement by the posterior approach—aetiology and treatment. Hong Kong J Orthop Surg $7: 14-18$

B. Saccomanni $(\square)$

Orthopaedic and Traumatologic Surgery,

University of Chieti, Via dei Vestini,

66013 Chieti Scalo, Italy

e-mail: bernasacco@yahoo.it 